\title{
An architectural strategy for meta-scheduling in Inter-clouds
}

\author{
Nik Bessis ${ }^{1}$, Stelios Sotiriadis ${ }^{1}$, Florin Pop $^{2}$, Valentin Cristea ${ }^{2}$ \\ ${ }^{1}$ School of Computing \& Maths, University of Derby, Derby, United Kingdom \\ ${ }^{2}$ University "Politehnica" of Bucharest, Bucarest, Romania \\ 1'(n.bessis, s.sotiriadis)@derby.ac.uk, ${ }^{2}$ (valentin.cristea, florin.pop)@cs.pub.ro
}

\begin{abstract}
Inter-clouds as a recently emerged approach has been introduced as to expand the cloud capabilities and to challenge the level of the cloud elasticity in services. By facilitating an opportunistic environment, it aims to consistently realise a scalable resource provisioning setting that handles sudden variation in user demands. Herein we propose an architectural strategy for scheduling jobs in interenterprises with a particular focus to inter-clouds. The scheduling concept is based on the meta-computing paradigm, with the purpose of establishing a wide and decentralized policy control among various resources and resource owners. To this extend, we start with presenting an analysis of the cloud characteristics and inter-cloud requirements. Following this, we present an identification of the most critical issues when developing the strategy based on the functional requirements analysis. This has led to the development of a strategic plan which provides the architecture for an interoperable, efficient and flexible environment to support the inter-cooperation between heterogeneous inter-enterprises.
\end{abstract}

Keywords: Clouds, Inter-clouds, Inter-enterprises, Federated Clouds, Meta-schedulers, Community Aware Scheduling

\section{INTRODUCTION}

Cloud computing turn out to be one of the most promising computing paradigms emerged during the last years. This is because of its great capacity to offer scalable and rigid elastic services. In such systems, the vast computing resources, which reside to a remote location, offer on-demand low-priced flexible services, including hardware, software and developers' platforms. Although cloud computing scales resources of various vendors data-centre in a satisfactory level [13] yet there is a concern that when endusers number and/or resource demand increase, the capacity oriented clouds (e.g. data or storage clouds) will reach their maximum service equilibrium.

This has been emphasised in [2] which introduces and highlights the need for a wide cloud collaborative environment. A remarkable example as discussed in [4] is the case of the Amazon cloud vendor which the overall resource management mechanism gives the impression of an initial imbalanced resource structure. Specifically, Amazon data-centres don't support auto scaling of resources e.g. outsourcing services or end-user's requests to low utilized enterprises for offering a well-balanced setting. In addition, it allows users to select their desired cloud data-centre based on their geographically location, thus decreasing the flexibility and the quality of service as a whole.

For addressing such issues various authors e.g. [2][4][7] suggest that inter-clouds could be the next step in the cloud evolution. Originally, they suggest that the inter-clouds approach is the natural progress of global application delivery and conclude that the most important requirement is essentially the auto-scaling of resource. Having said that, the challenge is that the actual requirements are not known prior to the resource administrator decision. Thus, a more realistic proposed solution would be a transitory engagement of temporary risen requirements to the resource managers.

In this paper, we take the view and define inter-clouds of inter-collaborative and inter-cooperative enterprises as a temporal auto-scaling resource formation in which services and resource exchange happen among various clouds but also amongst other e-infrastructures as to augment service quality and provide a total satisfaction for a wide range of customer diverse requirements. Such e-infrastructures should include but not limited to clusters, grids, high performance and throughput computing.

In general, the inter-clouds approach expand the cloud capabilities in terms of services with the aim of achieving a wider distribution of resources, yet by retaining global resource utilization equilibrium among various resource pools. In such settings, one of the most important design issues for an inter-collaborative cloud is the resource scheduling strategy with respect to its local cloud data-centre scheduling plan. Specifically, the approach implies that a local data-centre should participate in the on-demand resource selection process at both local (intra-) and global (inter-) scale as well as manage the resource selection, demand allocation and queuing of user tasks at a local level by considering the characteristics of the actual system (centralized or decentralized) as well as the requirements of the desired scenario.

In our previous works [2][13] we have presented a comparative study of various schedulers. Specifically, we have discussed that the meta-computing paradigm, hence meta-scheduling, has proven to be the most appropriate solution, because of its great flexibility when handling the complex requirements of each inter-cooperative system. As discussed before, the automation level of resource management has been the most important topic of interclouds [4]. In view of that, meta-computing could offer the 
infrastructure for managing unforeseen dynamic situations e.g. resource real time responses and failures.

Consequently, this paper aims at identifying the critical issues for developing a strategic plan for scheduling job tasks in inter-cloud environments. Within this context, in Section II we present a discussion of the related works toward to inter-cloud environment. Section III addresses the characteristics of clouds and a mapping to inter-clouds requirements. Section IV contains an analysis of the functional requirements for inter-clouds based on our previous works [2], [13]. Section V presents the development of an architectural based strategic plan for inter-cloud scheduling decisions. Finally, Section VII summarises the paper and discusses our future steps.

\section{RELATED WORKS}

The inter-cloud as a term has been emphasised by the leading vendors in cloud services area such as HP, Intel, Yahoo, etc. [4]. It is noticeable that their state-of-the-art efforts have led to the establishment of a federation of collaborated clouds with joint initiatives. However, this vendor-oriented endeavour of inter-clouds has a specific control plane rather than a setting that it is based on future standards and open interfaces which are available to be shared in the academic community. In addition, knowledge sharing, experimentation and testing within their systems have been limited to the wide range of researchers.

In a different direction, the work of [1] suggests a blueprint of inter-clouds, including network protocol and format. The authors propose the concept of a cloud operated by one service provider to inter-operate with clouds operated by another, thus forming a federation of clouds. They suggest that inter-operable network protocols at the lower level must be presented inside and in-between of clouds in order to achieve dynamic workload migration. However, despite the fact that there aren't any experimental evidences, the lower level communication of inter-clouds is out of the focus of our present study.

In contrast to aforementioned works, the vision of interclouds as an inter-cooperative infrastructure including interenterprises has been introduced by [4] yet from a federated perspective. They suggest a utility-oriented federation of various cloud computing environments and conclude to a business model of system architecture including the most important elements (requirements) of inter-clouds in terms of services. These functional specific requirements are presented in section IV and target to highlight the most important features for the strategic architectural plan of section VI. However, it is essential to introduce the most important characteristics of clouds first and map characteristics to inter-enterprises, by focusing in interclouds generic requirements extracted from [2].

Having said that, our primary interest here is to design a scheduler for inter-cloud environments. On this basis, in [13] we have surveyed a number of highly dynamic schedulers applied within meta-computing environments, i.e. grids and we have discussed their most important characteristics. Then, the work in [2], derived from [13], focuses on the dynamicness of a meta-scheduling approach that forms the basis of our research. Specific highly dynamic schedulers applied within meta-computing environments have been elaborated and useful conclusions have been determined. After, we swift the focus of our study in analysing the functional requirements for inter-clouds scheduling. We achieved this by producing a meta-scheduling comparison for inter-clouds [2]. This has led to the identification of the most important features when developing a meta-scheduling strategic plan. Figure 1 illustrates the rationale of our approach.

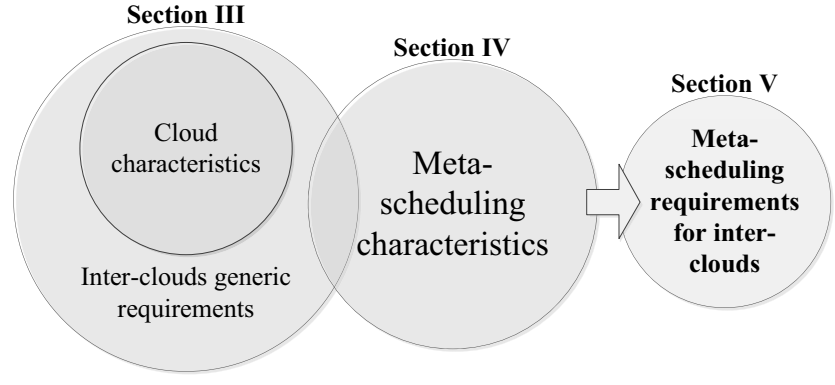

FIGURE 1: EXTRACTION OF META-SCHEDULING REQUIREMENTS

Specifically, the meta-scheduling requirements for interclouds are the intersection of the cloud characteristics, interclouds generic requirements and meta-scheduling characteristics. In the next section we present the first and second set of characteristics and requirements extracted from the literature.

\section{TOWARDS INTER-CLOUDS GENERIC REQUIREMENTS}

Herein, we discuss the generic characteristics (features) of clouds as well as the additional generic requirements for inter-clouds based on the business model of [4].

The characteristics of cloud computing are discussed extensively in [8] in which authors present a variety of related works in cloud definitions and cloud characteristics. It should be mentioned that cloud characteristics are bounded to the desired cloud conception, thus some suggest that it is more realistic to organise them according to specific scenarios [2]. For example, in Infrastructure-as-a-Service (IaaS) clouds the storage services are in the first plan, while the Platform-as-a-Service clouds (PaaS) are more softwareoriented. However, various works suggest definite characteristics of cloud computing as follows. [8] recommends that cloud characteristics are summarised in dynamic computing infrastructure; IT service centric approach; self-service based usage model; minimally or selfmanaged platform and consumption-based billing. Additionally, they suggest that cloud is more about a virtualized infrastructure; comprising of elastic/dynamic resource pools; providing self-oriented service access and usage based on user-tracking. [7] sum up other characteristics, e.g. automatic adaptation (dynamics); scalability; Service-Level Agreements (SLAs); resource optimisation, and virtualization. Finally, authors in [9] present a more generic view of the cloud features including loosely coupled view of resources; strong fault tolerant; business model; high security; virtualization and f) variation of resources. Certainly, all the aforementioned definitions 
and features are bounded to a specific scenario of the cloud environment. In our case scenario for a scheduling in interclouds, it is vital to identify those important characteristics that will become the fundamental aspects of inter-clouds. For that reason, in [2], we have presented a state-of-the-art review of eighteen meta-scheduling technologies. The purpose was the analysis of possible solutions for determining meta-scheduling to inter-enterprises including inter-clouds. Having said that, herein we intent to associate the characteristics of most meta-scheduling approaches to the aforesaid cloud features. Specifically, each meta-scheduling approach aims of addressing one or more of the cloud characteristics. Finally, through this correlation we conclude to the following characteristics which are important for our future cloud conception (scheduling in inter-clouds):

a) The dynamic-ness and automatic adaptation;

b) The virtualization of certain resources;

c) The resource optimization;

d) The loosely coupling of resources;

e) Self-management of resource;

f) Heterogeneity of resources.

To sum up, the above list forms equally a) the cloud characteristics for our case scenario and b) the basic requirements which are essential for the future inter-clouds meta-scheduler.

Next, we present the inter-clouds requirements as extracted from the business model presented in [4]. Specifically, the generic architectural strategy contains three key elements of an inter-cloud in the form of a federated collaboration namely a) the cloud coordination, b) the cloud broker, and c) the cloud exchange. At first, the cloud coordinator is responsible for domain specific enterprise of clouds. Also, the authors recommend that market-based trading mechanisms as well negotiation protocols should be implemented here. The basic functionalities including here are the scheduling and allocation, the market and policy engine, the application composition engine, the virtualization, the sensor and finally the discovering and monitoring of resource. Secondly the cloud broker acting on behalf of users identifies cloud service providers by utilizing the cloud exchange. The latter is responsible for keeping usage information e.g. cost, and updating the SLA policies. Indeed, this business model aims to fulfil needs of a commercialised-oriented federated clouds, rather than to a mutual agreed collaborative effort among inter-operable clouds and enterprises. In our view, inter-clouds should be an open initiatives environment that doesn't majorly purpose to the standardization of vendor clouds e.g. Amazon, Salesforces etc.

However, it is essential that the core requirements of inter-clouds e.g. use of virtualization technology, cost monitoring, exchanging of information among clouds for SLAs, discovery and monitoring, etc. are crucial to be defined and should be included to the general requirement model. For achieving our vision, we utilise and base our design to the above business model, including the market based mechanisms, and we focus our study to the scheduling and allocation part. Thus, extending schedulers to a metascheduling level by developing a meta-scheduling strategy it is expected that current job scheduling amongst clouds will be functioning and inter-cooperate at a federated cloud level. The next section presents the analysis of the meta-scheduling characteristics which are related to inter-clouds requirements.

\section{ANALYSIS OF FUNCTIONAL CHARACTERISTICS FOR INTER-CLOUDS META-SCHEDULING}

In this section we present a comparative study for identifying the crucial characteristics of meta-scheduling in inter-clouds for developing our architectural model. More specifically, we present properties of scheduling approaches of Clusters, HPC, Grids [3] and Clouds with the aim of extending the business model of [4] from a centralized to decentralized inter-clouds. Specifically, we achieve this by incorporating functionalities of the meta-computing paradigm with the purpose of establishing a decentralized and wide policy control among resources.

It should be mentioned that over the last decades, several computing architectures have been emerged with regard to their operational scenario. The most common of them in terms of large scale computing infrastructure and distribution of resources' workloads are the high performance computing (HPC), the grid and the cloud. We limit our study to these paradigms as the meta-scheduling scheme has been majorly applied to them with great success [5][12][14]. In addition, we present the cluster compuing paradigm as the simplest form of workload management. Specifically, the cluster computer is an aggregated view of linked computers working closely together forming a simple computer. However, this paradigm doesn't contain any distribution of resources.

Starting with, HPC, it is defined as an owner centric resource provisioning architecture in which resources are locally owned, and clients have private access to the owner organisation. The allocation happens with respect to users and projects and shared with respect to the workflows of a user or a group. The aim of HPC is to gain great computational power for solving complex problems, normally in a particular administrated environment.

In grid paradigm resources are locally and/or externally owned, thus wider administrated resources are observed. Members of the grid constitute a virtual organisation (VO) and have access to resources in a public manner [3]. Herein, heterogeneous resources, in terms of hardware and software could enter and leave the grid dynamically, while at the same time their capacity and performance might be altered. This makes the administration and scheduling a challenging issue.

In cloud computing resources can be externally or internally owned forming the public or the private cloud. The pay-on-demand model of clouds allows users to access resources, which size is dynamic growing using the virtualization technologies (virtual machines). This kind of dynamic sizing allows cloud a dynamic creation, migration and destruction of resources [8].

Each one of the aforementioned computing technologies described above has several advantages and drawbacks which have been studied by literature in detail and it is beyond the purpose of this study. However, as scheduling approaches - in addition to meta-scheduling - have been 
extensively studied in the aforesaid three paradigms, a draft comparison is presented in Table 1 in order to examine the key properties of HPC, grid and cloud computing.

Table 1: Properties of Clusters, HPC, Grid and Clouds in meta-scheduling

\begin{tabular}{|l|l|l|l|l|}
\hline $\begin{array}{l}\text { System } \\
\text { Properties }\end{array}$ & Cluster & HPC & Grid & Cloud \\
\hline $\begin{array}{l}\text { Resource } \\
\text { sharing }\end{array}$ & Limited & Limited & High & Limited \\
\hline $\begin{array}{l}\text { Geographically } \\
\text { distribution }\end{array}$ & $\begin{array}{l}\text { Not } \\
\text { applicable }\end{array}$ & Limited & High & Limited \\
\hline $\begin{array}{l}\text { Resource } \\
\text { heterogeneity }\end{array}$ & Limited & Limited & $\begin{array}{l}\text { Average to } \\
\text { high }\end{array}$ & $\begin{array}{l}\text { Limited } \\
\text { to } \\
\text { average }\end{array}$ \\
\hline $\begin{array}{l}\text { Structure } \\
\text { homogeneity }\end{array}$ & High & High & Limited & High \\
\hline Interoperability & $\begin{array}{l}\text { Not } \\
\text { applicable }\end{array}$ & $\begin{array}{l}\text { Not } \\
\text { applicable }\end{array}$ & Average & Limited \\
\hline Capacity & Fixed & Fixed & $\begin{array}{l}\text { Average to } \\
\text { high }\end{array}$ & High \\
\hline $\begin{array}{l}\text { Distribution of } \\
\text { workloads }\end{array}$ & $\begin{array}{l}\text { Not } \\
\text { applicable }\end{array}$ & $\begin{array}{l}\text { Not } \\
\text { applicable }\end{array}$ & High & Limited \\
\hline $\begin{array}{l}\text { Virtualization } \\
\text { support }\end{array}$ & Limited & Limited & $\begin{array}{l}\text { Limited to } \\
\text { average }\end{array}$ & High \\
\hline $\begin{array}{l}\text { Workload } \\
\text { management }\end{array}$ & High & High & High & Limited \\
\hline
\end{tabular}

Regarding the meta-scheduling concept in inter-clouds we examine the cloud properties as presented in table 1 to quantity the performance when implemented in large scale and uncertain size environment. Specifically we suggest that the system properties should be altered from a local resource management system (LRMS) to a decentralized one. Consequently, issues like interoperability and resource heterogeneity will be high. Those properties are summarised in table 2. It should be mentioned that we separate interclouds of clouds and enterprises with regards to their collaborative resources, e.g. inter-clouds of enterprises could contain grids and HPCs settings.

Table 2: Properties of inter-clouds in terms of meta-scheduling

\begin{tabular}{|l|l|l|}
\hline $\begin{array}{l}\text { System } \\
\text { Properties }\end{array}$ & $\begin{array}{l}\text { Inter-Clouds } \\
\text { of clouds }\end{array}$ & $\begin{array}{l}\text { Inter-Clouds of } \\
\text { enterprises }\end{array}$ \\
\hline Resource sharing & High & High \\
\hline Geographically distribution & High & High \\
\hline Resource heterogeneity & Average & High \\
\hline Structure homogeneity & Limited & High \\
\hline Interoperability & Average & High \\
\hline Capacity & High & High \\
\hline Distribution of workloads & Average & High \\
\hline Virtualization support & High & High \\
\hline Workload management & Average & High \\
\hline
\end{tabular}

In general, the meta-scheduling theme will originally define that each resource has a local and a meta-scheduler. Thus, jobs are directly submitted to a meta-scheduler and the last one decides to which local scheduler to relocate it. In the simplest of the cases, meta-schedulers query each other at regular intervals so as to collect current load data [11], and to find the site with the lowest load for transferring the job. This solution is the more advanced and complex as compared to centralized and hierarchical themes as it is more scalable and flexible. Specifically, the meta-scheduler has a partial and instantaneous knowledge of the environment. This partial knowledge based solution is usually related to the granularity of the system (the measure in which a system is broken down). For that reason, it is essential that resources of both inter-clouds of clouds and inter-clouds of enterprises are heterogeneous, may change unpredictably, are dynamic in nature and may vary in size.

To conclude, herein we have presented a comparative study for correlating the vital properties of meta-scheduling in HPC, grids, clouds and inter-clouds. Next, we present our architecture for meta-scheduling in inter-clouds by extending the work of [4] in which a centralized cloud exchanger is responsible for negotiation amongst clouds. Specifically, our proposed solution encompasses support for a total decentralized setting.

\section{ARCHITECTURAL STRATEGY FOR SCHEDULING IN INTER-CLOUDS AND INTER-ENTERPRISES}

Herein, we present a flexible architecture for incorporating the meta-scheduling approach and its algorithms for tasks with or without dependencies in intercloud environments. Finding the optimal procedures for meta-scheduling is important, especially in large scale distributed computing systems and complex applications for different research areas. As discussed in previous section, we consider that by utilizing the meta-scheduling approach as a solution for scheduling jobs in large scale and uncertain size environments will offer the required basic model for future inter-clouds scheduling algorithms to deal with high dynamics.

Starting with the traditional scheme, figure 2 shows the inter-clouds exchange model which contains the cloud exchanger, cloud coordinator and cloud broker.

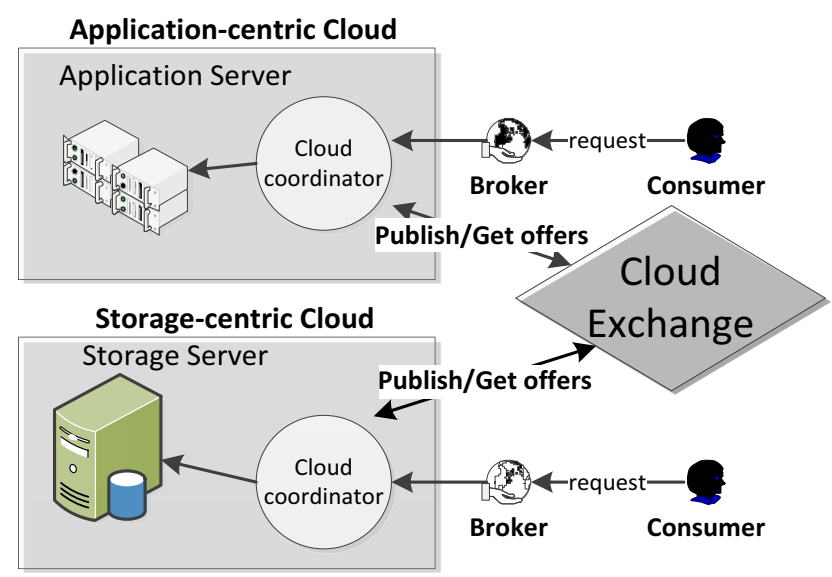

FIGURE 2: INTER-CLOUDS CLOUD EXCHANGE MEDIATOR

Each time a consumer requests for a service, the cloud broker negotiates with the cloud coordinator for required resources, cost and services. Then, the cloud coordinator decides whether the request could be completed by its own servers, or it will be published as an offer to the cloud exchange mediator. The cloud exchange will act in a centralized fashion by sending requests to each cloud coordinator. This market based mechanism will allow a 
federation of clouds to exchange services based on a common agreed financial model. However, as the size of the clouds for exchanging services grows, it is obvious that issues such as reliability, flexibility, bottleneck of requests and responses, and centralized failures will happen.

In the proposed inter-clouds environment, the decision for service exchanging happens throughout the metaexchanger. Figure 3 demonstrates the collaboration model among various clouds forming an inter-cloud of clouds. Specifically, each time a consumer requests for a service to each local broker, the request is redirected to the metacoordinator which contains the meta-scheduling policy of the cloud. The request is then forwarded to the local cloud coordinator (blue line) which contains the local policy of the LRMS. If the meta-coordinator decides that its LRMS is not capable of performing the job, the request is published to the meta-exchanger. After that, communication is redirected to other meta-exchangers (red lines) which they ask metacoordinators for resource availability. Finally, when a metaexchanger is selected policies are matched, and metacoordinators act as meta-schedulers by simple collaborating with each other directly (grey line). Finally, responds are going back to consumers using the broker component.

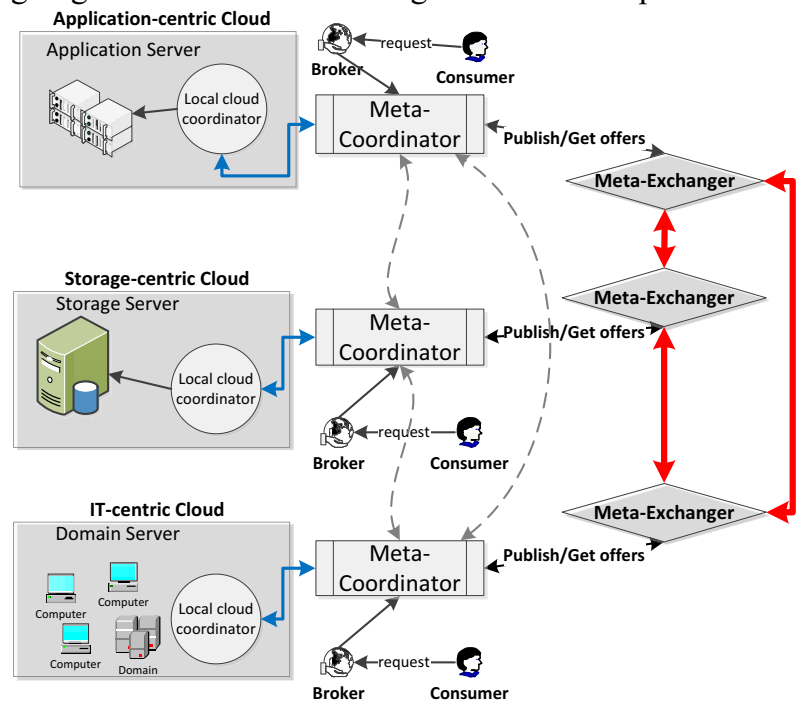

FIGURE 3: INTER-CLOUDS OF CLOUDS COLLABORATION ARCHITECTURE

In the case of an inter-cloud of enterprises (e.g. grids) the architecture is similar to the Figure 4 model. Specifically, each time a consumer requests for services, its broker redirects request to the meta-coordinator. The last one forwards the request to the local cloud coordinator (LRMS) and decides whether the job will be completed locally or will be forwarded to other meta-exchangers. In the second case, and when a meta-exchanger is selected the meta-schedulers collaborate directly for performing scheduling of jobs. It should be mentioned that parallel jobs could be fragmented in such way that various chunks of jobs could be executed by different LRMS of various meta-schedulers. Finally, a respond is sending back to the consumer through the broker component.
To conclude, meta-coordinators are placed on the top of the local system coordinator (LRMS) and are used to assign jobs to resources based on a great variety of criteria. However, a specification of each component of the proposed architecture is beyond the scope of the present work.

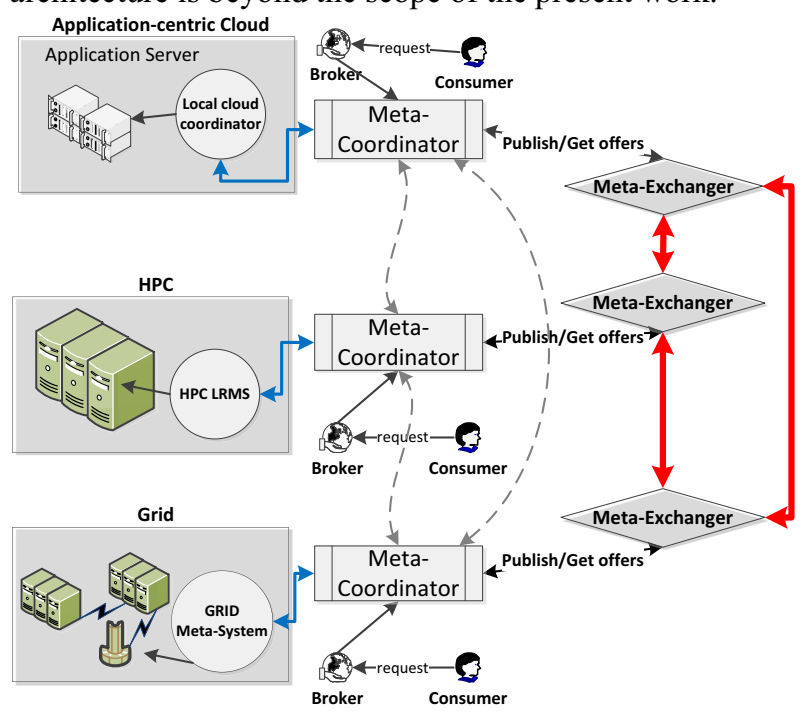

FIGURE 4: INTER-CLOUDS OF ENTERPRISES ARCHITECTURE

Therefore, in the next section we focus our study to the evaluation and simulation model for our future metascheduler for inter clouds.

\section{A. Evaluation model for inter-clouds scheduling}

In general, the identification of and comparison between different scheduling systems based on a set of evaluation criteria is not always applicable. This is because schedulers have been designed with a specific set of requirements in mind. In this proposal, we suggest those that are essential for our specific inter-clod scheduling scenario and these are summarized below.

a) Application Performance Promotion: This involves reviewing how well the applications can benefit from the deployment of the scheduling system.

b) System Performance Promotion: This concerns how well the whole system can benefit, like the utilization of resources is increased by, and how much the overall throughput gains)

c) Scheduling Efficiency: This includes that the scheduling system should introduce additional overhead as low as possible and the overhead introduced by the scheduling system may exist in the information collection, the mapping process, and the resources allocation.

d) Reliability: This concerns the level of fault-tolerance for large collection of loosely-coupled resources considering that the scheduler should handle such frequent resource failures.

e) Scalability: This includes that the scalable scheduling infrastructure should maintain good performance with not only increasing number of applications, but also increasing number of participating resources with heterogeneity [3]. 
f) Adaptability: This includes the level of flexibility to adapt to unforeseen situations.

Finally, we are particularly interested in the view that inter-clouds scheduling should also include a static and dynamic objective evaluation in an application and resource centric environment.

\section{B. Simulation model for inter-clouds scheduling}

The proposed simulation model is based on MONARC, a generic simulation framework designed for modeling large scale distributed systems. In general, when running an experiment the job object contains a number of parameters that are used to estimate the time needed for execution. The time needed by a job to complete a CPU-intensive operation is estimated based on a number of attributes such as the CPU power, memory and the processing time needed to complete the job. For the data processing jobs, these attributes depend on the type of data that the job works with (in the configuration file, the user can set this parameters for each data type used in the simulation). Once the CPU-intensive job starts processing the time needed to complete its operation is pre-computed. If another job starts executing on the same processing unit before the first one completes, then an interrupt mechanism is used to handle the re-estimation of the time needed for both jobs [6]. The time needed for an I/O intensive job (for example, a data transfer handling type of job) is based on the mechanism provided by the network model. In this case again an interrupt mechanism is used to simulate the competition for bandwidth usage of concurrent data transfer jobs [10]. Within the job model the user can define new jobs starting from the basic behaviour provided.

\section{CONCLUSION}

This work continues the work of [2] [13] aiming at the development of an architectural strategy for inter-clouds meta-scheduling. Prior to this, we suggested that the most important features of clouds and large scale systems are the dynamic-ness and automatic adaptation, the virtualization of certain resources, the resource optimization, the loosely coupling of resources, the self-management of resource, and the heterogeneity of resources. Based on that, we have proposed an architecture that models the collaboration among various clouds in two cases, the inter-clouds of clouds and inter-clouds of enterprises. Both solutions could offer significant improvements for developers who want to create decentralized management inter-clouds. Finally, we have suggested a basic evaluation model (scheduling objectives) and a simulation model for inter-clouds. The next step of the research study is the development of a state-ofthe-art meta-scheduler for inter-clouds of clouds and enterprises. In addition we aim of evaluating the benchmark results by comparing the performance of three highly dynamic meta-scheduling approaches as derived from [2] by implementing them in a specific simulation environment.

\section{ACKNOWLEDGEMENTS}

The first author would like to thank UNITE FP7248583 for their secondment support.

\section{REFERENCES}

[1] Bernstein, D.; Ludvigson, E.; Sankar, K.; Diamond, S.; Morrow, M.; "Blueprint for the Intercloud - Protocols and Formats for Cloud Computing Interoperability," Internet and Web Applications and Services, 2009. ICIW '09. Fourth International Conference on , vol., no., pp.328-336, 24-28 May 2009

[2] Bessis, N., Sotiriadis, S., Cristea, V., Pop, F., Towards inter-cloud schedulers: Modelling Requirements for Enabling Meta-Scheduling in Inter-Clouds and Inter-Enterprises, Third International Conference on Intelligent Networking and Collaborative Systems (INCOS 2011), Fukuoka, Japan, Nov 30 - Dec 22011 \{To appear\}

[3] Bessis, N. (ed, 2009), Grid Technology for Maximizing Collaborative Decision Management and Support: Advancing Effective Virtual Organizations, IGI Publishing, ISBN: 978-1-60566-364-7

[4] Buyya, R., Ranjan, R., and Calheiros, R. N., (2010) InterCloud: Utility-Oriented Federation of Cloud Computing Environments for Scaling of Application Services, Algorithms and Architectures for Parallel Processing (2010), Volume: 6081/2010, Issue: LNCS 6081, Publisher: Springer, Pages: 13-31

[5] Christodoulopoulos, K., Sourlas, V., Mpakolas, I., and Varvarigos, E., A comparison of centralized and distributed meta-scheduling architectures for computation and communication tasks in Grid networks, Computer Communications, Volume 32, Issues 7-10, 28 May 2009, Pages 1172-1184, ISSN 0140-3664

[6] Dobre, C., Stratan, C., Cristea, V., "Realistic simulation of large scale distributed systems using monitoring", 7th International Symposium on Parallel and Distributed Computing, ISPDC'08, (ISI, ISBN: 9780-7695-3472-5), pp. 434-438, Krakow, Poland, July 1-5, 2008.

[7] GICTF White Paper, 2010 Global Inter-Cloud Technology Forum, Use Cases and Functional Requirements for Inter-Cloud Computing, August 2010, 9, Available at: http://www.gictf.jp/doc/GICTF_Whitepaper_20100809.pdf, accessed at: $02 / 07 / 2011$

[8] Gong, C., Liu, J., Zhang, Q., Chen, H., and Gong, Z., 2010. The Characteristics of Cloud Computing. In Proceedings of the 2010 39th International Conference on Parallel Processing Workshops (ICPPW '10). IEEE Computer Society, Washington, DC, USA, 275-279.

[9] Huang, Y., Bessis, N., Norrington, P., Kuonen, P., and Hirsbrunner, B., Exploring decentralized dynamic scheduling for grids and clouds using the community-aware scheduling algorithm, Future Generation Computer Systems, In Press, Accepted Manuscript, Available online 13 May 2011, ISSN 0167-739X

[10] Istin, M., Visan, A., Pop, F., Dobre, C., and Cristea, V. 2010. NearOptimal Scheduling Based on Immune Algorithms in Distributed Environments. In Proceedings of the 2010 International Conference on Complex, Intelligent and Software Intensive Systems (CISIS '10). IEEE Computer Society, Washington, DC, USA, 439-444.

[11] Legrand, I. C., Dobre, C., Voicu, R., Stratan, C.,Cirstoiu, C., Musat, L., A Simulation Study for T0/T1 Data Replication and Production Activities", in Proc. of the 15th International Conference on Control Systems and Computer Science, Ed. Politehnica Press, Bucharest, Romania, 2005, pp. 131-135, ISBN: 973-8449-89-8, ISBN: 9738449-91-x.

[12] Pop, F., Dobre, C., Stratan, C., Costan, A., and Cristea, V. 2010. Dynamic meta-scheduling architecture based on monitoring in distributed systems. Int. J. Autonomic Comput. 1, 4 (December 2010), 328-349.

[13] Sotiriadis, S., Bessis, N., and Antonopoulos, N., Towards inter-cloud schedulers: A survey of meta-scheduling approaches, Sixth International Conference on P2P, Parallel, Grid, Cloud and Internet Computing, Barcelona, Spain, Oct 26-28 2011 \{To appear\}

[14] Xhafa, F., and Abraham, A., Computational models and heuristic methods for Grid scheduling problems, Future Generation Computer Systems, Volume 26, Issue 4, April 2010, Pages 608-621, ISSN 0167-739X 